\title{
Sphingolipids Are Required for Mammalian Epidermal Barrier Function Inhibition of Sphingolipid Synthesis Delays Barrier Recovery After Acute Perturbation
}

Walter M. Holleran, Man Mao-Qiang, Wen Ni Gao, Gopinathan K. Menon, Peter M. Elias, and Kenneth R. Feingold Departments of Dermatology and Medicine, University of California School of Medicine; and Dermatology and Medical Services, Veterans Administration Medical Center, San Francisco, California 94121

\begin{abstract}
Stratum corneum lipids comprise an approximately equimolar mixture of sphingolipids, cholesterol, and free fatty acids, arranged as intercellular membrane bilayers that are presumed to mediate the epidermal permeability barrier. Prior studies have shown that alterations in epidermal barrier function lead to a rapid increase in cholesterol and fatty acid synthesis which parallels the early stages of the repair process. Despite an abundance of indirect evidence for their role in the barrier, the importance of sphingolipids has yet to be demonstrated directly. Whereas sphingolipid synthesis also increases during barrier repair, this response is delayed in comparison to cholesterol and fatty acid synthesis (Holleran, W. M., et al. 1991. J. Lipid Res. 32:1151-1158). To further delineate the role of sphingolipids in barrier homeostasis, we assessed the impact of inhibition of sphingolipid synthesis on epidermal barrier recovery. A single topical application of $\beta$-chloro-L-alanine ( $\beta$-CA), an irreversible inhibitor of serine-palmitoyl transferase (SPT), applied to acetone-treated skin of hairless mice resulted in: (a) $>75 \%$ inhibition of SPT activity at $30 \mathrm{~min}(P<0.001)$; (b) a global decrease in sphingolipid synthesis between 1 and $3 \mathrm{~h}(P$ $<0.02)$; (c) reduction of epidermal sphingolipid content at $18 \mathrm{~h}$ $(P<0.01) ;(d)$ delayed reaccumulation of histochemical staining for sphingolipids in the stratum corneum; and $(e)$ reduced numbers and contents of lamellar bodies in the stratum granulosum. Finally, despite its immediate, marked diminution of sphingolipid synthesis, $\beta$-CA slowed barrier recovery only at late time points $(>6 \mathrm{~h})$ after acetone treatment. This inhibition was overridden by coapplications of ceramides (the distal SPT product), indicating that the delay in repair was not due to nonspecific toxicity. These studies demonstrate a distinctive role for epidermal sphingolipids in permeability barrier homeostasis. (J. Clin. Invest. 1991.88:1338-1345.) Key words: sphingolipids - serine palmitoyl transferase - epidermal barrier $\bullet \boldsymbol{\beta}$ chloro-alanine
\end{abstract}

\section{Introduction}

Stratum corneum lipids comprise a distinctive mixture, lacking in phospholipids, but enriched in ceramides, cholesterol, and free fatty acids (1-5). Although lipids are known to be critical for the epidermal permeability barrier, the role of specific lipid species is still the subject of intense study. Recent studies have shown that both cholesterol and fatty acid synthesis are required for permeability barrier homeostasis (6-10). An

Address correspondence and reprint requests to Dr. Walter M. Holleran, Dermatology Service (190), Veterans Administration Medical Center, 4150 Clement Street, San Francisco, CA 94121.

Received for publication 17 May 1991.

The Journal of Clinical Investigation, Inc.

Volume 88, October 1991, 1338-1345 immediate burst in both epidermal cholesterol and fatty acid synthesis occurs after barrier disruption, which normalizes over $6 \mathrm{~h}$ in parallel with the rapid, early phase of recovery $(6,8)$. Moreover, artificial restoration of the barrier by occlusion with water vapor-impermeable membranes after barrier disruption, blocks the expected burst in both cholesterol and fatty acid synthesis (6-8). Furthermore, the burst in cholesterol synthesis is paralleled by increases in both the total activity and activation state of 3-hydroxymethyl glutaryl CoA (HMG CoA) reductase (10), the rate-limiting enzyme of cholesterol synthesis (11). These increases also are prevented when barrier function is artificially restored by occlusion (10). Finally and most importantly, topical application of the HMG CoA reductase inhibitor, lovastatin, delays both barrier repair and the return of stainable cholesterol to the stratum corneum after acetone treatment (9).

Whereas these studies clearly demonstrate the importance of cholesterol for barrier homeostasis, the role of sphingolipids is still based primarily upon indirect evidence. First, sphingolipids account for up to $40 \%$ of the stratum corneum lipid weight $(1-5,12)$; second, among all of the esterified stratum corneum lipids, they are the principal repository of long chain, saturated fatty acids C26:0-C32:0 unique to the epidermis (5, $13,14)$; third, the long chain, saturated species are substituted by shorter chain, $N$-acyl species $(<\mathrm{C16})$ in marine mammals (cetaceans), who display much less stringent barrier requirements (15); fourth, they are the principal carriers of the essential fatty acid, linoleic acid, which is required for normal barrier function $(14,16-22)$; and fifth, only solvents that remove sphingolipids (as well as neutral lipids) from the stratum corneum are capable of abrogating the permeability barrier (23). Despite this impressive body of indirect evidence, the role of sphingolipids in epidermal barrier function is only now beginning to be studied directly. Recently, we demonstrated that barrier disruption with acetone, tape stripping, or in essential fatty acid deficiency (EFAD), ${ }^{1}$ resulted in increased sphingolipid synthesis (24), paralleling an increase in activity of serine palmitoyl transferase (SPT), the rate-limiting enzyme of sphingolipid synthesis $(25,26)$. Both of these alterations also were prevented by occlusion (24), providing initial direct evidence for a link between sphingolipid synthesis and barrier recovery. However, in contrast to cholesterol and fatty acid synthesis, sphingolipid synthesis did not increase until after the barrier had largely recovered, suggesting that de novo synthesis may not be required for the rapid, early phase of barrier recovery (24).

To elucidate further the role of sphingolipids in epidermal barrier homeostasis, we examined here the dynamics of epider-

1. Abbreviations used in this paper: $\beta$-CA, $\beta$-chloro-L-alanine; EFAD, essential fatty acid deficient; SPT, serine-palmitoyl transferase; TEWL, transepidermal water loss. 
mal barrier repair in the presence or absence of $\beta$-chloroalanine $(\beta-\mathrm{CA})$, a suicide inhibitor of SPT (27). We report here that: $(a)$ inhibition of sphingolipid synthesis by $\beta$-CA has no effect on the early stages of barrier recovery, but instead delays the late stages of barrier repair; $(b)$ this delay in recovery is associated with inhibition of epidermal lamellar body formation and secretion, with delayed reaccumulation of sphingolipids in the epidermis; and finally, $(c)$ simultaneous topical coapplication of ceramides, the predominant distal product of SPT in the epidermis (1-3), overrides the inhibitory effects of $\beta-\mathrm{CA}$ on barrier recovery, demonstrating the specificity of the link between barrier recovery and enzyme activity. Together, these studies indicate that epidermal sphingolipid synthesis is required for barrier homeostasis.

\section{Methods}

Materials. Reagent-grade organic solvents, $\beta$-chloro-L-alanine, pyridoxal phosphate, dithiothreitol, palmitoyl-CoA, and standards for high performance thin layer chromatography (HPTLC) (including sphingomyelin, sphingosine, ceramides III and IV, and cerebrosides I and II) were obtained from Sigma Chemical Co (St. Louis, MO). Hepes buffer was purchased from Fisher Scientific Co. (Santa Clara, CA); $\left[{ }^{3} \mathrm{H}\right] \mathrm{H}_{2} \mathrm{O}$ (sp act $5.0 \mathrm{Ci} / \mathrm{ml}$ ) was from New England Nuclear (Boston, MA); [G$\left.{ }^{3} \mathrm{H}\right] \mathrm{L}$-serine was from ICN Radiochemicals (Irvine, CA). Bio-Rad protein reagent and bovine serum albumin were obtained from Bio-Rad Laboratories (Richmond, CA). HPTLC plates (silica gel 60) were purchased from E. Merck (Darmstadt, FRG) (lot No. 5641).

Animals and functional studies. Hairless male mice $(\mathrm{Hr} / \mathrm{Hr})$ were purchased from Jackson Laboratory (Bar Harbour, ME) or Simonsen Laboratories (Gilroy, CA). They were fed Purina mouse diet and water ad lib, and all were 8-12-wk-old at the time of study. Transepidermal water loss (TEWL) was measured at various time points with an electrolytic water analyzer (Meeco; Harrington, PA), as described previously (24).

Experimental groups. Murine epidermis (five or six mice in each experiment) was gently treated with acetone-soaked cotton balls until TEWL rates exceeded $300 \mathrm{ppm} / 0.5 \mathrm{~cm}^{2}$ per $\mathrm{h}\left(3.0 \mathrm{mg} / \mathrm{cm}^{2}\right.$ per h). Immediately after acetone treatment, cohorts of animals were left untreated on one flank; other groups received either: (a) $400 \mu \mathrm{g}$ of $\beta$-chloroalanine $(10 \mathrm{mg} / \mathrm{ml}$ in a propylene glycol/ethanol vehicle; 70:30, vol/ vol); or ( $b$ ) $\beta$-chloroalanine (as above) plus an equal concentration of bovine ceramides (III \& IV) in the same vehicle; or (c) vehicle alone, to an area $\sim 2.5 \times 2.5 \mathrm{~cm}$. TEWL was measured $2,4,6,12,14,18,24$, and $36 \mathrm{~h}$ after treatment. At various time points samples were taken for enzyme assay, lipid synthesis studies, analysis of lipid content, histochemistry, and electron microscopy (see below).

SPT assay. Samples were obtained for SPT assay $30 \mathrm{~min}, 6 \mathrm{~h}$, and $18 \mathrm{~h}$ after topical applications. Our procedure for isolation and quantitation of SPT from murine epidermis has been described in detail elsewhere $(24,28)$. Briefly, epidermis was separated from dermis after incubation of whole skin samples in 10 mM EDTA (in PBS-calcium-magnesium-free), homogenized on ice using a Polytron PCU2 (Kinematica $\mathrm{GmbH}$, Lucerne, Switzerland) in Hepes $50 \mathrm{mM}, \mathrm{pH} 7.4$, containing 10 $\mathrm{mM}$ EDTA, $5 \mathrm{mM}$ DTT, and $0.025 \mathrm{M}$ sucrose, followed by sonication using a Fisher Sonic Dismembranator (model 300; Artec Systems Corp., Farmingdale, NY), as previously described $(10,24)$. Differential centrifugation $\left(4^{\circ} \mathrm{C}\right)$ was performed and the microsomal pellet $(100,000 \mathrm{~g} ; 60 \mathrm{~min})$ was resuspended in storage buffer containing 50 mM Hepes (pH 7.4), 5 mM EDTA, 5 mM DTT, and 20\% glycerol (vol/vol), and stored at $-70^{\circ} \mathrm{C}$ until use.

Our assay for SPT activity is modified from Williams et al. (26), as recently described (28). Briefly, the assay buffer contained $100 \mathrm{mM}$ Hepes, pH 8.3, 5.0 mM DTT, and 2.5 mM EDTA, while the reaction mixture contained $50 \mu \mathrm{M}$ pyridoxal phosphate (PyrP), $150 \mu \mathrm{M}$ palmitoyl-coenzyme A (PCoA), 1.0 mM [G- $\left.{ }^{3} \mathrm{H}\right]-\mathrm{L}-$ serine (sp act $45-50,000$ $\mathrm{dpm} / \mathrm{nmol}$ ), and 50 to $100 \mu \mathrm{g}$ of microsomal protein in $0.1 \mathrm{ml}$ total assay volume. The reaction was initiated by simultaneous addition of PCoA and $\left[{ }^{3} \mathrm{H}\right]$-L-serine, incubated at $37^{\circ} \mathrm{C}$ for $10 \mathrm{~min}$, and terminated by the addition of $0.2 \mathrm{ml}$ of $0.5 \mathrm{~N}$ ammonium hydroxide. The reaction product, 3-ketodihydrosphinganine (3KDS), was isolated as described previously (28), and counted using a Beckman LS-1800 scintillation counter (Beckman Instruments, Inc., Fullerton, CA). Protein content was determined using the Bradford method (29). Enzyme specific activity was expressed as pmol of $3 \mathrm{KDS}$ formed per min per mg of microsomal protein.

Sphingolipid synthesis. The effect of $\beta$-CA on sphingolipid synthesis was assessed in vivo after intraperitoneal injections of $\left[{ }^{3} \mathrm{H}^{3} \mathrm{H}_{2} \mathrm{O}(20\right.$ $\mathrm{mCi} / 0.2 \mathrm{ml}$ ) administered $1 \mathrm{~h}$ after acetone treatment and $\beta$-CA application. $2 \mathrm{~h}$ after the injection, blood samples were taken and the animals were killed. Whole skin was excised $\left(\sim 8 \mathrm{~cm}^{2}\right)$ from each flank (acetone-treated and -untreated sides), heated for $60 \mathrm{~s}\left(60^{\circ}\right)$, and the epidermis separated from the dermis with gentle scraping. Samples were blotted dry, weighed, minced, and immediately placed into screwcap glass test tubes containing Bligh-Dyer solution (30), and total lipid extracts were prepared, as described previously (3). The lipid components were separated by HPTLC as previously described (24), visualized by Woods light fluorescence after 8-anilino-1-naphthalene-sulfonic acid staining (5), identified by cochromatography with known standards, scraped into scintillation vials, and counted by liquid scintillation spectrometry. Total incorporation of tritium into sphingolipids was obtained by combining the ceramides, glycosphingolipids, sphingomyelin, and sphingosine base fractions. Using the specific activity of $\left[{ }^{3} \mathrm{H}^{\mathrm{H}} \mathrm{H}_{2} \mathrm{O}\right.$ in serum samples from each animal, results were presented as $\mu$ mol product formed per $2 \mathrm{~h} / \mathrm{g}$ of epidermal wet weight, as described previously $(6-8,24,31)$.

Lipid content. 6 and $18 \mathrm{~h}$ after treatment (see above), epidermal sheets were obtained from hairless mouse skin by exposure to dry heat $\left(60^{\circ} \mathrm{C}\right.$ for $\left.60 \mathrm{~s}\right)$. Lipids were extracted from the epidermal sheets as described previously $(3,24)$, dried, weighed, and stored at $-70^{\circ} \mathrm{C}$ in chloroform until use. For separation and quantitation of individual sphingolipid species, we employed a modification of the method of Ponec et al. (32). Extracts were applied to precleaned $10 \times 20-\mathrm{cm}$ HPTLC plates, $0.5 \mathrm{~cm}$ from lower edge of plate and fractionated sequentially in a horizontal developing chamber (Camag; Muttenz, Switzerland) in the following solvent systems: (a) chloroform/methanol/ acetic acid (190:9:1, vol), twice to $10 \mathrm{~cm}$; and then redeveloped in chloroform/methanol/acetone $(76: 20: 4, \mathrm{vol})$ to $30 \mathrm{~cm}$. Plates were dried for $5 \mathrm{~min}$ at $40^{\circ} \mathrm{C}$, cooled for $2 \mathrm{~min}$, then equilibrated in the tank for $5 \mathrm{~min}$ between each solvent run. After the final solvent run, the dried plates were dipped in charring solution (1.5\% cupric sulfate in acetic acid/sulfuric acid/orthophosphoric acid/distilled $\mathrm{H}_{2} \mathrm{O}$ (50:10:10:30, vol), dried $\left(40^{\circ} \mathrm{C}, 10 \mathrm{~min}\right)$, and then charred at $180^{\circ} \mathrm{C}$ for $5 \mathrm{~min}$. The plates were scanned with a variable wavelength scanning densitometer (Camag) and the lipid fractions quantitated by comparison to known standards run in duplicate simultaneously for each function. Integration was performed using CATS II software (Camag).

Histochemistry. Fresh, full thickness skin samples were obtained before treatment, immediately after acetone treatment, and at various time points after applications of $\beta$-CA or vehicle to acetone-treated skin. Each sample was snap-frozen and stored at $-70^{\circ} \mathrm{C}$ until $6 \mu \mathrm{m}$ frozen sections were prepared on a Universal Scientific, Inc. cryostat (Atlanta, GA). Nile red was applied for visualization of neutral versus polar lipids, as described previously $(23,33)$. Sections then were examined and photographed with a Leitz Ortholux II microscope (E. Leitz, Inc., Rockleigh, NJ), equipped for epifluorescence.

Electron microscopy. At various time points after treatment with the protocols described above, skin biopsies were minced to $<0.5$ $\mathrm{mm}^{2}$, and fixed in modified Karnovsky's fixative overnight. Samples were then postfixed in both ruthenium tetroxide, using a recently described protocol (34), and $2 \%$ aqueous osmium tetroxide both containing $1.5 \%$ potassium ferrocyanide. After fixation, all samples were dehydrated in graded ethanol solutions, and embedded in an Epon-epoxy mixture (35). Thin sections were examined, with or without further 
contrasting with lead citrate, in a Zeiss 10A electron microscope (Carl Zeiss, Inc., Thornwood, NY), operating at $60 \mathrm{kV}$.

Statistical analysis. Statistical evaluation of data was performed using either a two-tailed Student's $t$ test or paired $t$ test.

\section{Results}

Effect of $\beta$-chloroalanine on epidermal serine palmitoyl transferase activity. Previous studies have shown that abundant SPT activity is present in murine epidermis (28), and that this activity is increased after barrier disruption (24). In addition, $\beta$-CA potently inhibits SPT in cultured human keratinocytes (28). In these studies, we first assessed the impact of topical $\beta$-CA application on epidermal SPT activity in order to insure that the drug reaches sites of sphingolipid synthesis in the nucleated layers beneath the stratum corneum. Direct application of $\beta$ CA $\left(400 \mu \mathrm{g} / 7 \mathrm{~cm}^{2}\right)$ to intact skin resulted in no inhibition of enzyme activity, presumably due to limited access of $\beta$-CA across intact stratum corneum. However, when the barrier was first disrupted with acetone before $\beta$-CA application, a $77 \%$ inhibition of SPT activity was observed 30 min after treatment $(P<0.001)$ (Table I), indicating that delivery of $\beta$-CA to the epidermis occurred.

We next determined the duration of the action of $\beta$-CA in vivo after a single topical application to acetone-treated skin. Hence, in addition to the 30 -min time point, we measured SPT activity at 6 and $18 \mathrm{~h}$ after acetone plus $\beta$-CA $\left(400 \mu \mathrm{g} / 7 \mathrm{~cm}^{2}\right)$. The 6-h time point was selected because our prior studies showed that maximal stimulation of SPT activity occurs at this time after acetone disruption (24). As expected, a statistically significant increase $(50 \%)$ in total SPT activity $(P<0.005)$ occurred $6 \mathrm{~h}$ after acetone treatment (Table I). Moreover, application of the saline vehicle alone to acetone-treated sites did not inhibit this expected increase in SPT activity at $6 \mathrm{~h}$. In contrast, treatment with $\beta$-CA resulted in a significant inhibition of SPT activity in comparison to both normal and vehicletreated skin $(P<0.01$ and 0.001 , respectively). By $18 \mathrm{~h}$ after acetone treatment, SPT activity in the vehicle-treated sites returns to levels comparable to the untreated controls. Similarly, activity in the $\beta$-CA-treated sites had also returned to normal. These results show that a single application of $\beta$-CA produces

Table I. SPT Activity after Acetone Barrier Disruption and Topical $\beta$-Chloro-L-Alanine Treatment

\begin{tabular}{|c|c|c|c|c|}
\hline \multirow[b]{3}{*}{ Time } & \multicolumn{4}{|c|}{ Specific activity* } \\
\hline & \multirow{2}{*}{$\frac{\text { Untreated }}{\text { Normal }}$} & \multicolumn{2}{|c|}{ Acetone-treated } & \multirow{2}{*}{$\begin{array}{l}\text { Percentage } \\
\text { activity }^{*}\end{array}$} \\
\hline & & Plus vehicle & Plus $\beta$-CA & \\
\hline $30 \mathrm{~min}$ & $\begin{array}{c}74.8 \pm 8.6 \\
(n=8)\end{array}$ & $\begin{array}{c}68.9 \pm 7.5 \\
(n=3)\end{array}$ & $\begin{array}{c}15.8 \pm 7.3^{\S} \\
(n=4)\end{array}$ & 22.9 \\
\hline $6 \mathrm{~h}$ & $\begin{array}{c}87.1 \pm 9.1 \\
(n=9)\end{array}$ & $\begin{array}{c}123.3 \pm 13.1^{11} \\
(n=9)\end{array}$ & $\begin{array}{c}57.5 \pm 13.5^{\prime} \\
(n=9)\end{array}$ & 46.6 \\
\hline $18 \mathrm{~h}$ & $\begin{array}{c}83.6 \pm 7.4 \\
(n=6)\end{array}$ & $\begin{array}{c}86.0 \pm 5.0 \\
(n=6)\end{array}$ & $\begin{array}{c}82.7 \pm 9.1^{* *} \\
(n=6)\end{array}$ & 96.2 \\
\hline
\end{tabular}

* Specific activity $=\mathrm{pmol} / \mathrm{min}$ per $\mathrm{mg}+\mathrm{SD}(n) .{ }^{\ddagger}$ Percentage of activity $=($ activity [plus $\beta-C A] /$ activity [vehicle] $) \times 100 .{ }^{8} P<0.001$ versus vehicle or versus untreated normal. " $P<0.005$ versus untreated normal. ' $P<0.01$ versus untreated normal and $P<0.001$ versus acetone-treated plus vehicle. ${ }^{* *} P=$ NS.
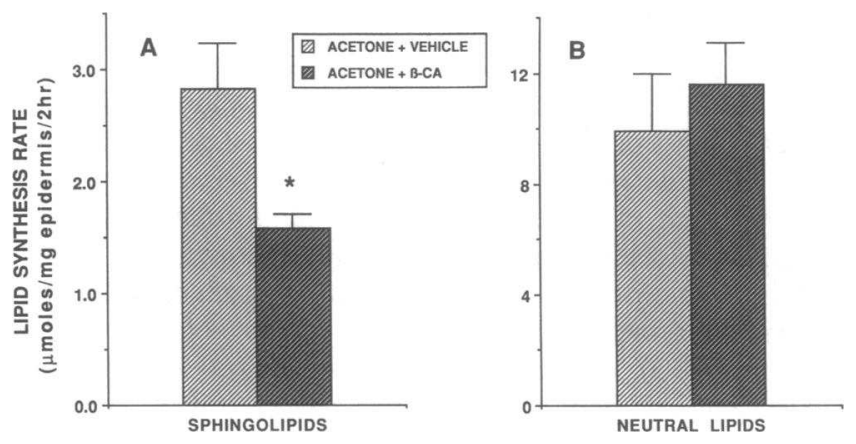

Figure 1. Inhibition of sphingolipid synthesis after acetone treatment and a single topical application of $\beta-C A .\left[{ }^{3} \mathrm{H}\right] \mathrm{H}_{2} \mathrm{O}$ was injected intraperitoneally $1 \mathrm{~h}$ after barrier disruption, and incubated for $2 \mathrm{~h}$. Results represent the mean rate of synthesis $( \pm$ SEM) of animals and are compared with animals treated with acetone plus vehicle alone. $(A)$ $\beta$-CA-inhibited sphingolipid synthesis by $40 \%, P<0.02$; (B) $\beta$-CA had no effect on total neutral lipid synthesis.

an immediate, profound inhibition of SPT activity, which is sustained for several hours, with a return of enzyme activity to normal levels by $18 \mathrm{~h}$.

Effect of $\beta$-chloroalanine on epidermal sphingolipid synthesis. In addition to addressing the influence of $\beta$-CA on SPT activity, we also assessed its ability to inhibit sphingolipid synthesis in vivo. A significant decrease in the total incorporation of $\left[{ }^{3} \mathrm{H}\right] \mathrm{H}_{2} \mathrm{O}$ into sphingolipids occurred between $1-3 \mathrm{~h}(>40 \%$; $P<0.02$ ) after $\beta$-CA application to acetone-treated skin (Fig. 1 $A)$. This degree of inhibition was comparable to the extent of SPT inhibition over the same time period (see Table I). Values for acetone plus vehicle-treated controls did not differ from untreated, normal controls (data not shown), indicating that the observed inhibition of sphingolipid synthesis could not be attributed to the acetone treatment and/or vehicle effects. Finally, the specificity of the $\beta$-CA effect on sphingolipid synthesis is further supported by the observation that ${ }^{3} \mathrm{H}^{3} \mathrm{H}_{2} \mathrm{O}$ incorporation into neutral lipids was unchanged in the presence of $\beta$-CA (Fig. $1 B$ ).

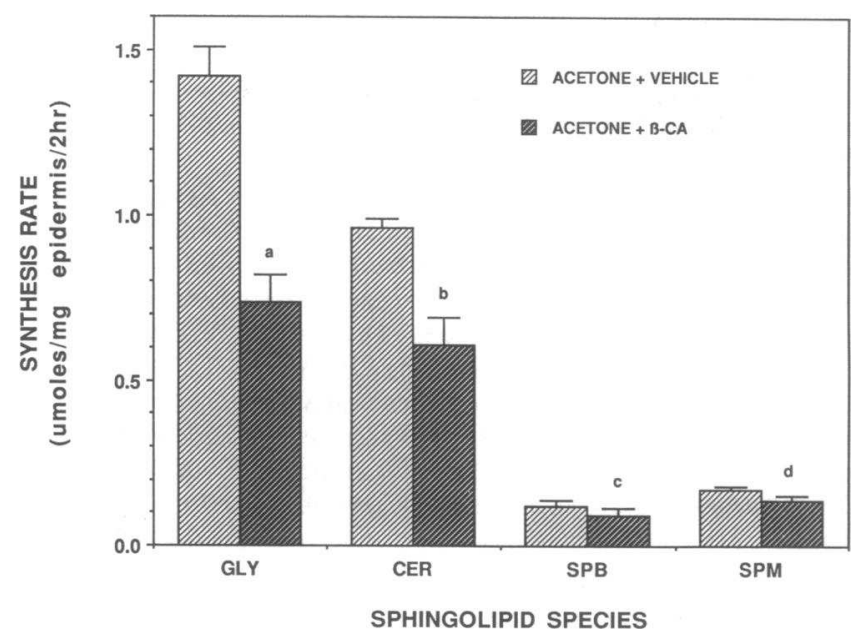

Figure 2. Effect of $\beta$-CA on synthesis of specific sphingolipid species. Animals were treated as described above (Fig. 1), and results are presented as mean ( $\pm \mathrm{SEM}) ; G L Y$, glycoceramides; $S P M$, sphingomyelin; $C E R$, ceramides; $S P H$, sphingosine base. ${ }^{a, b . c, d} P \leq 0.001,0.005,0.05$, 0.05 , respectively. 


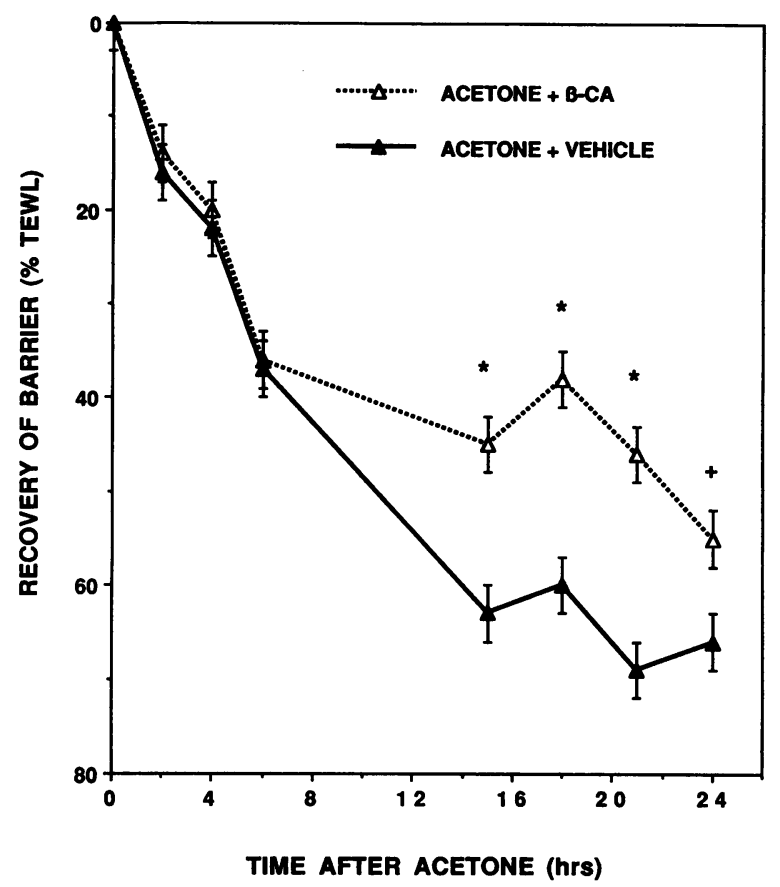

Figure 3. Recovery of epidermal barrier (TEWL) with time after acetone treatment $\pm \beta$-CA. Animals were treated with acetone and an immediate topical application of $\beta$-CA or vehicle alone. TEWL rates are plotted as the percentage recovery of TEWL. Each point represents the mean of at least five TEWL measurements on each of five or more mice ( $>25$ readings). ${ }^{*+} P$ values of $<0.01$, and $\leq 0.05$, respectively.

After ascertaining the global decrease in sphingolipid synthesis, we next examined the generation of each major sphingolipid class after application of $\beta$-CA to acetone-treated skin (Fig. 2). Each class was significantly decreased when compared with vehicle-treated controls, with the synthesis of glycosphingolipids reduced by $48 \%(P<0.001)$, while ceramide synthesis was decreased by $37 \%(P<0.005)$. Synthesis of the two minor sphingolipid classes, sphingomyelin and total sphingoid base, was reduced by 19 and $22 \%$, respectively $(P \leq 0.05)$. These results show that a single topical application of $\beta$-CA to ace-

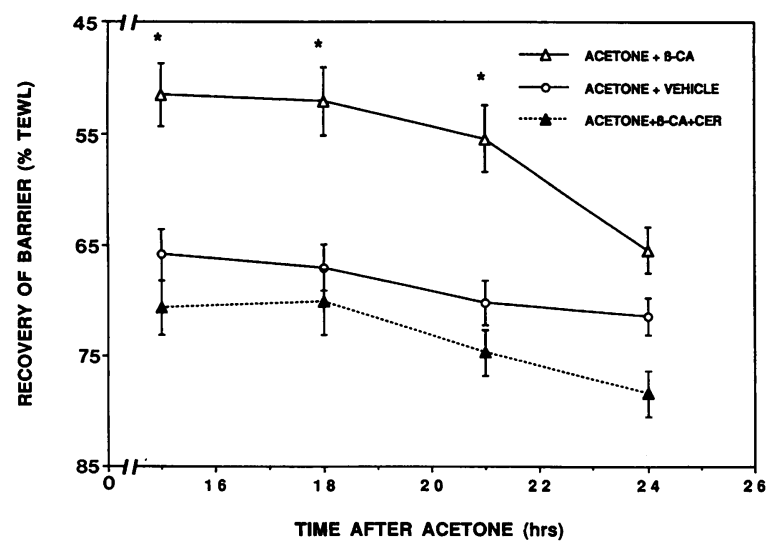

Figure 4. Ceramide override of $\beta$-CA. Coapplication of ceramide with $\beta$-CA to acetone-treated epidermis $(-\Lambda-)$ normalized the recovery of TEWL when compared with $\beta$-CA alone $(-\Delta-)$. Acetone-treated epidermis with vehicle alone is also shown $(-0-) .{ }^{*} P<0.005 ; n$ $\geq 15$ for each value.

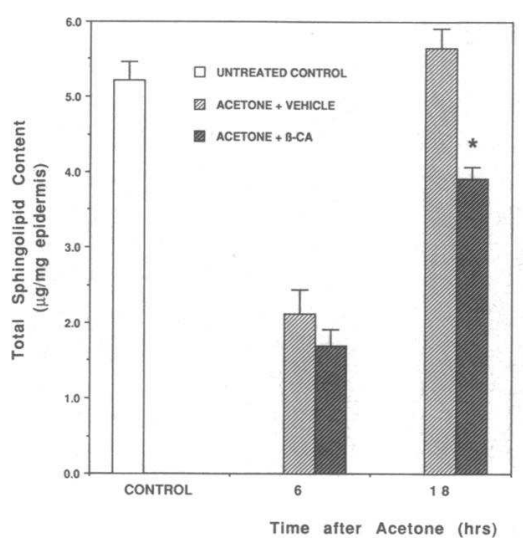

Figure 5. Epidermal sphingolipid content. Animals were treated with acetone and immediate application of $\beta$-CA or vehicle. At 6 and $18 \mathrm{~h}$, epidermal samples were obtained, and lipid extractions performed as described in Methods. Results are presented as micrograms sphingolipid (mean \pm SEM), per gram epidermal wet weight ( $n \geq 4$ for each). ${ }^{*} P \leq 0.01$.

tone-treated skin results in a decrease in the synthesis of sphingolipids.

Influence of $\beta$-chloroalanine treatment on barrier recovery. To determine whether inhibition of sphingolipid synthesis influences barrier recovery, we next measured barrier recovery in acetone-treated animals, treated with either $\beta$-CA or vehicle alone. As noted in numerous prior studies, barrier recovery after acetone treatment (with or without vehicle) appears to be a two-phase process, with $50-60 \%$ recovery occurring over the first $6 \mathrm{~h}$, and a relatively slow rate of recovery requiring $35-40 \mathrm{~h}$ thereafter (Fig. 3) (8). As seen in Fig. 3, no differences were observed in barrier recovery between the vehicle- and $\beta$-CAtreated groups over the first $6 \mathrm{~h}$. However, at later time points, $\beta$-CA treatment produced a significant delay in the rates of barrier recovery $(P<0.01$ at 15,18 , and $22 \mathrm{~h} ; P<0.05$ at $24 \mathrm{~h})$. At $36 \mathrm{~h}$, the difference between the two groups was not statistically significant (data not shown).

To determine both the specific relationship of these results to sphingolipid synthesis, and to rule out nonspecific toxicity as the cause of delayed recovery, we simultaneously applied equal amounts of $\beta$-CA and ceramide, the major end product of epidermal sphingolipid synthesis. When ceramides were coapplied with $\beta$-CA, the $\beta$-CA-induced inhibition of barrier recovery that occurs between 15 and $24 \mathrm{~h}$ was reversed; i.e., TEWL levels were the same as those observed in vehicle-treated skin (Fig. 4). These data demonstrate: first, that topical $\beta$-chloroalanine produces a delay in the later slow phase of barrier repair after acetone treatment; and second, that the delay is not due to nonspecific toxicity, because ceramides override the $\beta$-chloroalanine-induced alteration; and third, that the delay in recovery can be attributed to inhibition of sphingolipid synthesis.

Sphingolipid content. To determine whether the delayed barrier recovery could be due to decreased sphingolipid formation, we next compared total epidermal sphingolipid content in $\beta$-CA versus vehicle-treated controls. At $6 \mathrm{~h}$ after acetone treatment, both the vehicle- and $\beta$-CA-treated epidermis contained significantly less sphingolipid than did untreated control epidermis (Fig. 5), reflecting lipid removal by acetone (23). However, at this time point, $\beta$-CA-treated epidermal lipid content was not significantly different from the vehicle control. In contrast, by $18 \mathrm{~h}$, the $\beta$-CA-treated epidermis displayed significantly less sphingolipid content, suggesting that the delay in the later stages of barrier recovery with $\beta-\mathrm{CA}$ is due to decreased sphingolipid generation. 

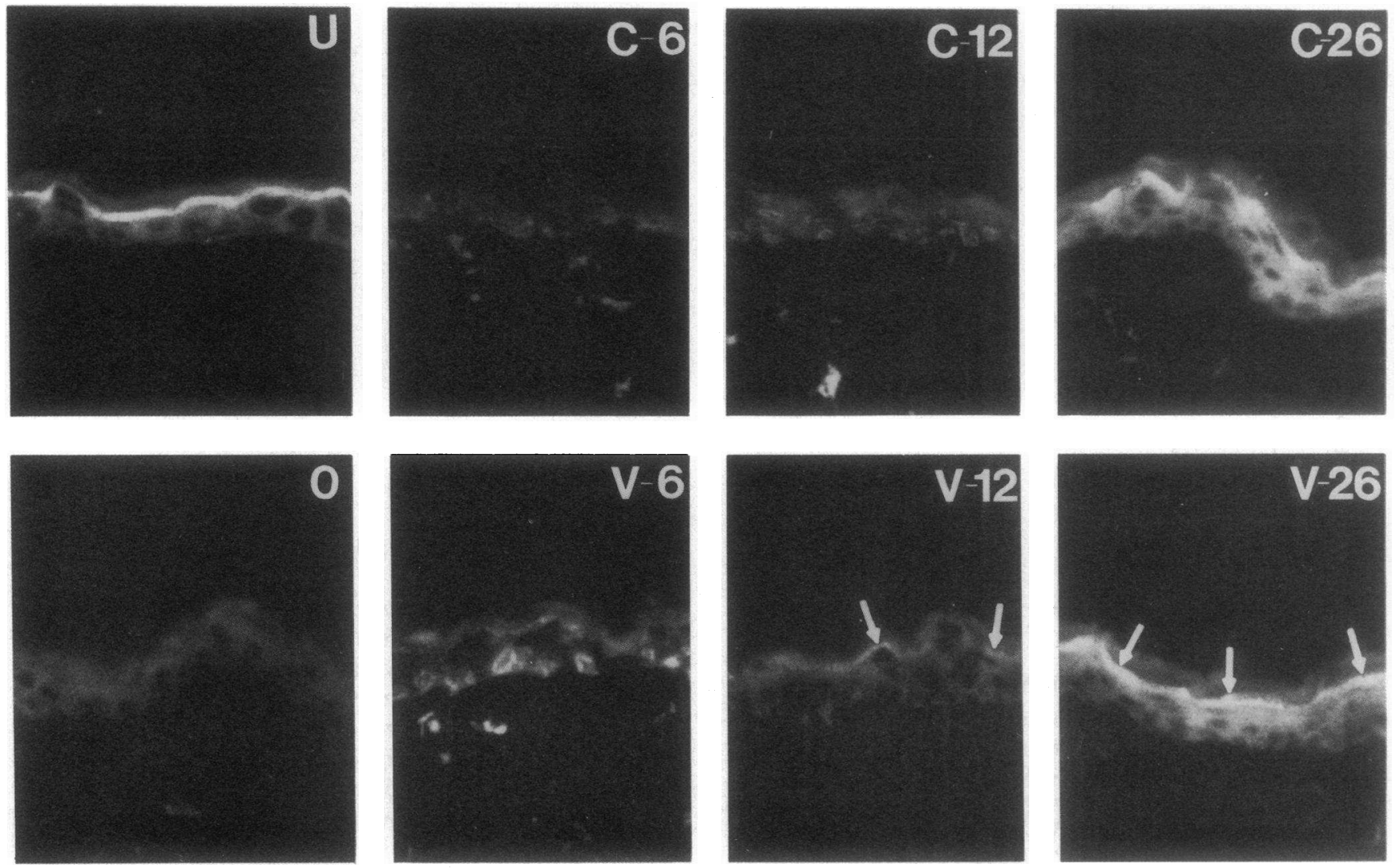

Figure 6. Reappearance of stainable polar lipids after $\beta$-CA versus vehicle-treatment. Untreated epidermis $(U)$ reveals an intense band of polar lipids at the stratum granulosum-stratum corneum interface, which is absent immediately after acetone treatment $(O)$. In vehicle-treated epidermis $(\mathrm{V})$, the slight appearance of stainable polar lipids is apparent as early as $12 \mathrm{~h}$ (arrows), but by $26 \mathrm{~h}$, the intensity of staining in the stratum corneum equals that of untreated skin. In $\beta$-CA-treated samples $(C)$, polar staining is also decreased from normal at 6 and $12 \mathrm{~h}$. However, by $12 \mathrm{~h}$, the $\beta$-CA-treated samples showed less polar lipid staining than the vehicle-treated controls. By $26 \mathrm{~h}$, a generalized increase in polar lipid staining is evident in the $\beta$-CA-treated samples, but the intense band of staining seen in the vehicle-treated sample is not present. All panels, magnification, 750 .

Effect of $\beta$-chloroalanine on the reappearance of stratum corneum sphingolipids. In order to further delineate whether the $\beta$-CA-induced delay in barrier repair reflects a delayed redeposition of sphingolipids in the stratum corneum, we next examined nile red-stained frozen sections of skin from untreated normals versus acetone-treated samples immediately before and after treatment with $\beta$-CA or vehicle alone. Because phospholipids are absent in mammalian stratum corneum (15), orange-red staining of polar lipids by nile red can be ascribed to sphingolipids (33). A comparison of sections immediately after acetone-treatment versus untreated controls showed that acetone treatment depleted the stratum corneum of polar lipids (Fig. 6, $O$ versus $U$ ). By $6 \mathrm{~h}$, occasional foci of polar lipid staining were apparent in vehicle-treated samples, but in general both the $\beta$-CA- and vehicle-treated samples revealed little or no polar lipid staining in the stratum corneum (Fig. 6, $V$-6 versus $C$-6). By $12 \mathrm{~h}$, the staining pattern in $\beta$-CAtreated samples diverged more clearly from vehicle-treated samples with distinctively less polar lipid staining apparent in the $\beta$-CA-treated group (Fig. 6, $C$-12 versus $V$-12). A difference between $\beta$-CA- and vehicle-treated samples was still present, although less prominent, at $26 \mathrm{~h}$ (Fig. 6, $C-26$ versus $V$-26). These results demonstrate that: $(a)$ after acetone-induced disruption of the barrier, sphingolipids are rapidly removed and then return to the stratum corneum in parallel with the recovery of barrier function; and $(b)$ the late onset, $\beta$-CA-induced delay in barrier repair correlates with a delayed reappearance of sphingolipids in the stratum corneum.

Electron microscopy. As described previously (9), acetone treatment produced an immediate secretion of lamellar bodies, from the outermost cells of the stratum granulosum, which is accompanied by cytosolic depletion of these organelles. By $3 \mathrm{~h}$, nascent lamellar bodies were seen in vehicle-treated samples, which largely displayed normal internal structure (Fig. $7 A$ ). Moreover, these nascent organelles continued to be secreted into the intercellular spaces, so that the stratum granulosumstratum corneum interface is engorged with newly secreted lamellar material. Likewise, the epidermis of animals treated with acetone followed by $\beta$-CA demonstrated abundant secreted lamellar material at the stratum granulosum-stratum corneum interface at $3 \mathrm{~h}$ (Fig. $7 \mathrm{~B}$ ). However, decreased numbers of nascent organelles are present in the cytosol at $3 \mathrm{~h}$, and those that could be identified lack typical, disc-like internal contents (Fig. 7 B). Even at $18 \mathrm{~h}$, nascent lamellar bodies could be identified solely in the lower layers of the stratum granulosum, with the cytosol of upper granular cells still largely devoid of these organelles (Fig. $7 \mathrm{C}$ ). As a result, the intercellular spaces at the stratum granulosum-stratum corneum interface 


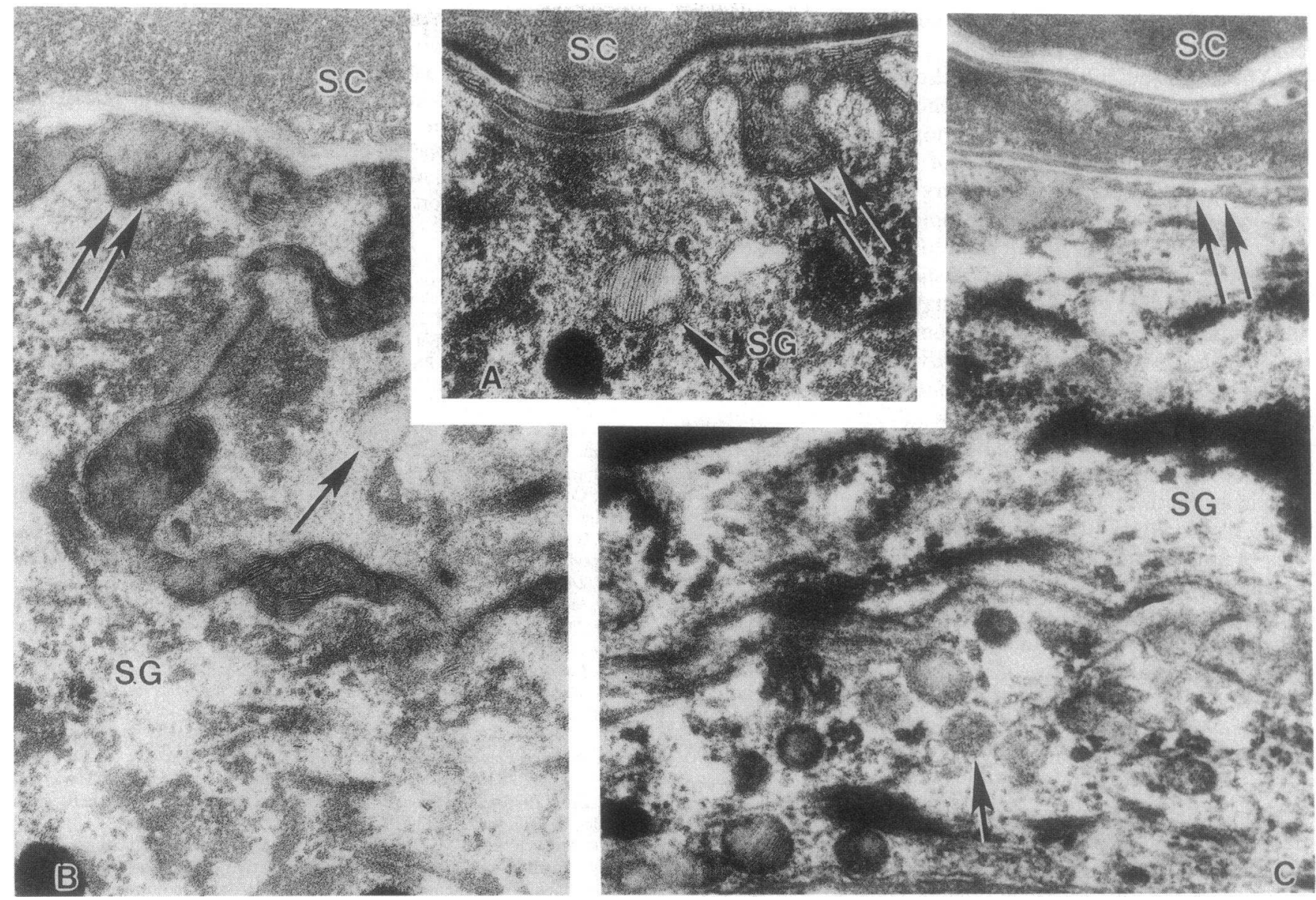

Figure 7. Ultrastructure of $\beta$-CA versus vehicle-treated epidermis at 3 and $18 \mathrm{~h} .(A) 3 \mathrm{~h}$ after acetone treatment, vehicle-treated controls reveal nascent lamellar bodies with normal internal, disc-like contents (single arrow). The quantity of secreted material at the stratum granulosum $(S G)$-stratum corneum $(S C)$ interface also appears normal (double arrows). $(B) 3 \mathrm{~h}$ after a single $\beta$-CA application to acetone-treated skin, the SG-SC interface appears replete (double arrows), but the few lamellar bodies that are present intracellularly display an abnormal appearance (single arrow). (C) 18 hours after a single $\beta$-CA application, the cytosol of outer SG cells is still devoid of lamellar bodies, and the SG-SC interface appears depleted of secreted lamellar material (double arrows). Although numerous lamellar bodies are seen in the lower SG, many still display abnormal internal structure (single arrow). $A, B, C$, magnification, 80,000, 62,000, 32,000, respectively.

appeared depleted of lamellar material in comparison with 3or 6-h samples (Fig. $7 \mathrm{C}$, top). Finally, although the number of lamellar bodies in the lower granular layer appears normal at $18 \mathrm{~h}$, the content of many of these organelles is still abnormal, as in 3-h $\beta$-CA-treated samples (Fig. $7 C$; c.f., Fig. 7 B). These results show that $\beta$-CA applications impede the reformation, packaging, and secretion of lamellar bodies in the outer epidermis, and cause distinct abnormalities in the internal structure of these organelles.

\section{Discussion}

Prior studies from our laboratory have demonstrated the link between epidermal cholesterol synthesis and permeability barrier function (6-10). Recent studies also have demonstrated that increased sphingolipid synthesis occurs when barrier function is abrogated (24). Yet, despite the wealth of compelling indirect evidence (see Introduction), a requirement for sphingolipids for mammalian barrier function has never been demonstrated directly. The results presented here demonstrate that inhibition of sphingolipid synthesis is accompanied by a significant delay in the expected rates of barrier recovery after acute disruption. $\beta$-CA is an irreversible inhibitor of SPT (27), the enzyme that catalyzes the initial condensation of L-serine and palmitoyl-CoA $(25,26)$, the first committed step in sphingolipid synthesis. We have shown here that $\beta$-CA inhibits SPT when applied topically, and that this inhibition is associated with both a specific and a global inhibition of synthesis of all sphingolipid species. Moreover, inhibition of sphingolipid synthesis is accompanied by a decrease in sphingolipid content, demonstrated both by a decrease in the return of polar lipid staining to $\beta$-CA-treated stratum corneum and by direct biochemical analysis. The specific requirement for sphingolipids is further demonstrated by the override studies, where coapplications of ceramides, the predominant sphingolipids in mammalian stratum corneum $(1-5,12)$, reverse $\beta$-CA-induced inhibition. Because ceramides applied alone to acetone-treated skin actually aggravate barrier function (Mao-Qiang, M., K. R. Feingold, and P. M. Elias, manuscript in preparation), the corrective effect of ceramides also can not be ascribed to a bulk effect of the lipid alone. Finally, the override studies show that 
the effect of $\beta$-CA on the barrier can not be ascribed to nonspecific toxicity.

The results described here and elsewhere resemble recent, analogous studies with inhibitors of cholesterol synthesis (9). In these prior studies, we showed that topical applications of lovastatin, a competitive inhibitor of HMG CoA reductase, slowed the initial stages in the recovery of barrier function after acetone treatment (9). Moreover, topical coapplications of either cholesterol or mevalonate with lovastatin normalized the rate of barrier repair. Finally, just as shown here biochemically and histochemically for $\beta-\mathrm{CA}$, lovastatin applications delayed the return of cholesterol to the stratum corneum (9).

Yet, these studies reveal distinctive differences in the kinetics of cholesterol versus sphingolipid replenishment after acute disruption of the barrier. While inhibition of cholesterol synthesis produces an immediate delay in barrier repair that returns to normal by $6 \mathrm{~h}$ (9), the impact of inhibition of sphingolipid synthesis on barrier function is delayed, appearing first at $12 \mathrm{~h}$. Moreover, whereas the synthesis of cholesterol increases immediately after barrier disruption, returning to near normal levels by six $h(8,31)$, we recently reported that the increase in sphingolipid synthesis is also delayed (24). These differences can not be explained by differences in access of drug to target enzyme, because we have shown that both HMG CoA reductase (9) and SPT are inhibited rapidly after topical application of lovastatin and $\beta-\mathrm{CA}$, respectively. Moreover, the differences can not be explained by a different origin of these two lipid pools, because the epidermal lamellar body is enriched in both cholesterol and sphingolipids $(12,36)$, and both would be deposited simultaneously as this secretory organelle undergoes exocytosis. Possible explanations for the dissimilarity of the results with the two inhibitors include differences in the size, turnover rate, or destinations of the two lipid pools; e.g., sufficient preformed sphingolipids may be present to mediate the early stages of barrier repair, while the cholesterol pool may be smaller or multifunctional, and therefore more readily depleted. For example, a substantial proportion of lamellar bodyderived free sterols may be diverted to other membrane functions or metabolic destinations (e.g., vitamin D), leaving a smaller pool of free sterols available for barrier repair.

These studies demonstrate a requirement for sphingolipid synthesis in addition to cholesterol and fatty acids for the maintenance of epidermal barrier homeostasis. Together these species form the membrane bilayers that mediate epidermal barrier function (reviewed in reference 37 ). It is likely that a deficiency of any of these species will result in abnormal membrane structures, with a reduced capacity to impede transepidermal water flux. This view is supported not only by the inhibitor studies with lovastatin $(9,38)$ and $\beta$-CA (these studies), but also by data from selected metabolic disorders where altered proportions of one or more of these species is accompanied by abnormal barrier function. In neutral lipid storage disease (39), Refsum's Disease (40), and in rodent $\operatorname{EFAD}(16,17,21,22)$, free sterol and sphingolipid content are reportedly normal, but fatty acid content is defective, and in the last case, elevated TEWL rates are a prominent feature. Thus, it is likely that a mixture of sphingolipids, cholesterol, and free fatty acids form the membrane bilayer system that is required to maintain epidermal barrier function. Further studies will be required to elucidate how cholesterol, free fatty acid, and sphingolipid synthesis are coordinated by barrier requirements.

\section{Acknowledgments}

The authors gratefully acknowledge Mr. Yutaka Takagi for assistance with lipid quantitation and Mr. Bil Chapman and Sally Michael for manuscript preparation.

This work was supported by National Institutes of Health grants AR-19098, AR-39448, AR-39639, and the Medical Research Service, Veterans Administration.

\section{References}

1. Gray, G. M., and H. J. Yardley. 1975. Different populations of pig epidermal cells: isolation and lipid composition. J. Lipid Res. 16:441-447.

2. Elias, P. M., B. E. Brown, P. Fritsch, P. Goerke, G. M. Gray, and R. J. White. 1979. Localization and composition of lipids in neonatal mouse stratum granulosum and stratum corneum. J. Invest. Dermatol. 73:339-348.

3. Lampe, M. A., A. L. Burlingame, J. A. Whitney, M. L. Williams, B. E Brown, E. Roitman, and P. M. Elias. 1983. Human stratum corneum lipids: characterization and regional variations. J. Lipid Res. 24:120-130.

4. Cox, P., and C. A. Squire. 1986. Variations in lipids in different layers of porcine epidermis. J. Invest. Dermatol. 87:741-744.

5. Bowser, P. A., and R. J. White. 1985. Isolation, barrier properties, and lipid composition of stratum compactum, a discrete region of the stratum corneum. Br. J. Dermatol. 112:1-14.

6. Menon, G. K., K. R. Feingold, A. H. Moser, B. E. Brown, and P. M. Elias. 1985. De novo sterologenesis in the skin. II. Regulation by cutaneous barrier requirements. J. Lipid Res. 26:418-427.

7. Feingold, K. R., B. E. Brown, S. R. Lear, A. H. Moser, and P. M. Elias. 1986. The effect of essential fatty acid deficiency on cutaneous sterol synthesis. $J$. Invest. Dermatol. 87:588-691.

8. Grubauer, G., K. R. Feingold, and P. M. Elias. 1987. Relationship of epidermal lipogenesis to cutaneous barrier function. J. Lipid Res. 28:746-752.

9. Feingold, K. R., M. Q. Man, G. K. Menon, S. S. Cho, B. E. Brown, and P. M. Elias. 1990. Cholesterol synthesis is required for cutaneous barrier function in mice. J. Clin. Invest. 86:1738-1745.

10. Proksch, E., P. M. Elias, and K. R. Feingold. 1990. Regulation of 3-hydroxy-3-methylglutaryl-coenzyme A reductase activity in murine epidermis:modulation of enzyme content and activation state by barrier requirements. J. Clin. Invest. 85:874-882.

11. Rodwell, Y. W., K. C. Nordstrom, and J. H. Mitschelen. 1976. Regulation of HMG CoA reductase. Lipid Res. 14:1-74.

12. Wertz, P. W., D. T. Downing, R. K. Freinkel, and T. N. Traczyk. 1985. Sphingolipids of the stratum corneum and lamellar granules of fetal rat epidermis. J. Invest. Dermatol. 83:193-195.

13. Wertz, P. W., and D. T. Downing. 1983. Acylglucosylceramides of pig epidermis: structure determination. J. Invest. Dermatol. 24:753-758.

14. Bowser, P. A., D. H. Nugteren, R. J. White, U. M. T. Houtsmuller, and C. Prottey. 1985. Identification, isolation and characterization of epidermal lipids containing linoleic acid. Biochim. Biophys. Acta. 834:419-428.

15. Elias, P. M., G. K. Menon, S. Grayson, B. E. Brown, and S. J. Rehfeld. 1987. Avian sebokeratinocytes and marine mammal lipokeratinocytes: structural, lipid biochemical and functional considerations. Am. J. Anat. 180:161177.

16. Prottey, C. 1976. Essential fatty acids and the skin. Br. J. Dermatol. 94:579-587.

17. Elias, P. M., and B. E. Brown. 1978. The mammalian cutaneous permeability barrier: defective barrier function in essential fatty acid deficiency correlates with abnormal intercellular lipid deposition. Lab. Invest. 39:574-583.

18. Abraham, W., P. W. Wertz, and D. T. Downing. 1985. Linoleate-rich acylglucosyl ceramides of pig epidermis: structure determination by proton magnetic resonance. J. Lipid Res. 26:761-766.

19. Hamanaka, S., C. Asagami, M. Suzuki, F. Inagaki, and A. Suzuki. 1989. Structure determination of glucosyl- $\beta-1-\mathrm{N}$ ( $\omega$-O-linoleoyl)-acylsphingosines of human epidermis. J. Biochem. 105:684-690.

20. Elias, P. M., B. E. Brown, and V. A. Ziboh. 1980. The permeability barrier in essential fatty acid deficiency: evidence for a direct role of linoleic acid in barrier function. J. Invest. Dermatol. 74:230-233.

21. Houtsmuller, U. M. T., and A. van der Beck. 1981. Effects of topical applications of fatty acids in essential fatty acid deficiency. Prog. Lipid Res. 20:219-224.

22. Wertz, P. W., E. S. Cho, and D. T. Downing. 1983. Effects of essential fatty acid deficiency on the epidermal sphingolipids of the rat. Biochim. Biophys. Acta. 753:350-355.

23. Grubauer, G., K. R. Feingold, R. M. Harris, and P. M. Elias. 1989. Lipid content and lipid type as determinants of the epidermal permeability barrier. $J$. Lipid Res. 30:89-96. 
24. Holleran, W. M., K. R. Feingold, M. Q. Man, W. N. Gao, J. M. Lee, and P. M. Elias. 1991. Regulation of epidermal sphingolipid synthesis by permeability barrier function. J. Lipid Res. 32:1151-1158.

25. Braun, P. E., P. Morell, and N. S. Radin. 1970. Synthesis of C18- and C20-dihydrosphingosines, ketodihydrosphingosines, and ceramides by microsomal preparations from mouse brain. J. Biol. Chem. 245:335-341.

26. Williams, R. D., E. Wang, and A. H. Merrill. 1984. Enzymology of longchain base synthesis by liver: characterization of serine palmitoyl transferase in rat liver microsomes. Arch. Biochem. Biophys. 228:282-291.

27. Medlock, K. A., and A. H. Merrill. 1988. Inhibition of serine palmitoyl transferase in vitro and long-chain base biosynthesis in intact Chinese hamster ovary cells by beta-chloroalanine. Biochemistry. 27:7079-7084.

28. Holleran, W. M., M. L. Williams, W. N. Gao, and P. M. Elias. 1990 Serine-palmitoyl transferase from human neonatal keratinocytes and murine epidermis. J. Lipid Res. 31:1655-1661.

29. Bradford, M. M. 1976. A rapid and sensitive method for quantitation of microgram quantities of protein utilizing the principle of protein-dye binding. Anal. Biochem. 72:248-254.

30. Bligh, E. G., and W. J. Dyer. 1959. A rapid method of total lipid extraction and purification. Can. J. Biochem. Physiol. 37:911-917.

31. Feingold, K. R., B. E. Brown, S. R. Lear, A. H. Moser, and P. M. Elias. 1983. Localization of de novo sterologenesis in mammalian skin. J. Invest. Dermatol. 81:365-369.

32. Ponec, M., A. Weerheim, J. Kempenaar, A.-M. Mommaas, and D. H. Nugteren. 1988. Lipid composition of cultured human keratinocytes in relation to their differentiation. J. Lipid Res. 29:949-961.
33. Grubauer, G., P. M. Elias, and K. R. Feingold. 1989. Transepidermal water loss: the signal for recovery of barrier structure and function. J. Lipid Res. 30:323-333.

34. Hou, S. Y. E., A. K. Mitra, S. H. White, G. K. Menon, R. Ghadially, and P. M. Elias. 1991. Membrane structures in normal and essential fatty acid deficient stratum corneum: characterization by ruthenium tetroxide staining and x-ray diffraction. J. Invest. Dermatol. 96:215-223.

35. McNutt, N. S., and W. L. Crain. 1981. Quantitative electron microscope comparison of lymphatic nuclear contours in mycosis fungoides and in benign infiltrates in the skin. Cancer. 47:163-166.

36. Grayson, S., A. G. Johnson-Winegar, B. U. Wintroub, E. H. Epstein, Jr., and P. M. Elias. 1985. Lamellar body-enriched fractions from neonatal mice: preparative techniques and partial characterization. J. Invest. Dermatol. 85:289295.

37. Elias, P. M., and G. K. Menon. 1991. Structural and lipid biochemical correlates of the epidermal permeability barrier. Adv. Lipid Res. 24: In press.

38. Feingold, K. R., M. Mao-Qiang, E. Proksch, G. K. Menon, B. Brown, and P. M. Elias. 1991. The lovastatin-treated rodent: a new model of barrier disruption and epidermal hyperplasia. J. Invest. Dermatol. 96:201-209.

39. Elias, P. M., and M. L. Williams. 1985. Neutral lipid storage disease with ichthyosis: defective lamellar body contents and intercellular dispersion. Arch. Dermatol. 121:1000-1008.

40. Dykes, P. J., R. Marks, M. G. Davies, and D. J. Reynolds. 1987. Epidermal metabolism in heredopathia atactica polyneuritiformis (Refsum's disease). $J$. Invest. Dermatol. 70:126-129. 